\title{
Model based multi-wavelength spectrophotometric method for calculation of formation constants of phenanthrenequinone thiosemicarbazone complexes with some metallic cations
}

\author{
Naser Samadi ${ }^{a}$, Mina Salamati ${ }^{a}$ and Abdolhossein Naseri ${ }^{b}{ }^{* *}$
}

${ }^{a}$ Department of Chemistry, Faculty of Science, Urmia University, Urmia, Iran ${ }^{b}$ Department of Analytical Chemistry, Faculty of Chemistry, University of Tabriz, Tabriz, Iran

\section{H R O N I C L E}

Article history:

Received October 28, 2012

Received in Revised form

March 1, 2013

Accepted 8 March 2013

Available online

10 March 2013

Keywords:

Complex formation

Hard-modeling

Multi-wavelength

Spectrophotometry \begin{abstract}
A B S T R A C T
In traditional spectrophotometric determination of stability constants of complexation, it is necessary to find a wavelength at which only one of the components has absorbance without any spectroscopic interference of the other reaction components. In the present work, a simple multi-wavelength model-based method has been developed to determine stability constants for complexation reaction regardless of the spectra overlapping of components. Also, pure spectra and concentration profiles of all components are extracted using multi-wavelength model based method. In the present work spectrophotometric titration of several cationic metal ions with new synthetic ligand were studied in order to calculate the formation constant(s). In order to estimate the formation constants a chemometrics method, model based analysis was applied.
\end{abstract}

(C) 2013 Growing Science Ltd. All rights reserved.

\section{Introduction}

Quinonoidal compounds of natural or synthetic origin represent the second largest class of clinically approved anticancer agents ${ }^{1}$. Their cytotoxicities are explained on the basis of many mechanisms including intercalation, inhibition of DNA and RNA, breaking of DNA strands, alteration of cell membrane functions, and free radical mediated alkylation ${ }^{2}$. Metal complexes of hydroxy and aminonaphthoquinones, as well as ortho-benzoquinones, have been studied frequently ${ }^{3}$. Pierpont and coworkers have studied the coordination chemistry of 3,5-di-t-butylbenzoquinones extensively ${ }^{4}$ while Abakumov et al. have carried out investigations on the metallic compounds of

* Corresponding author. Tel.: (+98) 411 3393106, Fax: (+98) 4113340191

E-mail addresses: a_naseri@tabrizu.ac.ir (A. Naseri)

C 2013 Growing Science Ltd. All rights reserved. 
tetrachloro o-benzoquinone ligands ${ }^{5}$. Another potential ligand of the o-quinone series, viz 9,10phenanthrenequinone (PQ), is also known to form complexes with transition metal ions, but whose coordination compounds are comparatively less investigated. The propensity of metal chelation in PQ is found to be enhanced when one of the carbonyl oxygen is appended with a thiosemicarbazone functionality as in scheme $\mathbf{1}$ and the ligand is noted as a chromogenic reagent and indicator for chelatometric determination of metal ions. Our attention was drawn to PQ and it's derivatives because of their planar structure resembling the phenanthroline compounds which are capable of undergoing intercalating interactions with DNA resulting in cell antiproliferative activities ${ }^{6}$. A combination of such planar polycyclic compound with potent cytotoxic thiosemicarbazone pharmacophore has been shown to produce synergetic effects on the antiproliferative activity of the parent steroidal ligand.

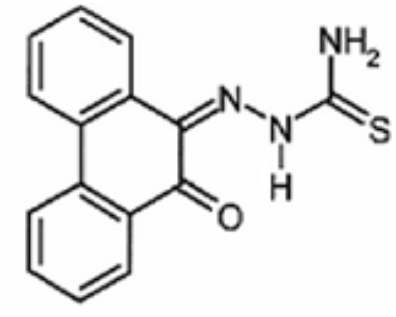

Scheme 1

The formation constants of complexes can be calculated using several techniques such as UV-Vis spectrophotometric method, NMR, and electrochemical methods ${ }^{7-14}$. The advantages such as simplicity, low cost and ease to use are the main reasons of variety application of potentiometric and spectrophotometric methods. Spectrophotometric methods have been utilized as an alternative to determine stability constants of complexes with large molar absorptivities because of its high sensitivity at concentrations of substance as low as $10^{-6} \mathrm{M}$. Nowadays, the spectrophotometric techniques remain largely used in this field because of the easy interpretation and handling of the spectral data. On the other hand, the conventional spectrophotometric methods use a discrete number of wavelengths that frequently are not enough to furnish the necessary information to resolve a system with severe spectra overlapping. In spite of the spectrophotometric method is rapid, cheap and simple, the direct spectrometric measurements show lack of specificity because the spectra are strongly overlapped. In such cases the chemometric techniques become an indispensable tool to overcome these problems ${ }^{15-19}$.

In any data analysis, a set of measured data from which one may try to extract useful information is dealt. Two different strategies are convenient in order to gain the formation constants of complexes. Model based analysis uses a primarily known model and model free analysis which uses a soft method to resolve the data. In both methods the aim was to achieve the complexation constants but additional information can also be extracted. For example the spectra of the included components can be acquired even in the presence of extreme overlapping. Also in the soft method several constraints, e.g. non-negativity, uni-modality and closure could be applied ${ }^{20-25}$.

In the present work the formation constants of several bivalent metal ions $\mathrm{Cu}$ (II), $\mathrm{Ni}$ (II), $\mathrm{Co}$ (II), $\mathrm{Zn}(\mathrm{II})$ with a new ligand, phenanthrenequinone thiosemicarbazone, were estimated using a model based analysis method.

\section{Experimental}

\subsection{Reagents}

The ligand phenanthrenequinone thiosemicarbazone (PQTSC), was synthesized according to previously published procedures ${ }^{26-28}$. The reactants for synthesis of PQTSC, 9, 10- 
phenanthrenequinone (PQ) and thiosemicarbazide, were purchased from Merck with the highest purity available and used without further purification. All other solvents and chemicals are commercially available (Merck) were used without purification.

\subsection{Methods}

Acetonitrile was used to preparation of metallic cations and ligand stock solutions. $2 \mathrm{~mL}$ of ligand solution $\left(10^{-5} \mathrm{M}\right)$ was transferred to a $3 \mathrm{~mL}$ quartz cell and was titrated by micro liter amounts of concentrated metal cation solutions (e.g. $0-150 \mu \mathrm{L}$ from $10^{-4} \mathrm{M}$ ). The solutions were thermostated at $25{ }^{\circ} \mathrm{C}$. An example for the change of the absorbance due to complex formation is given in Fig. 1 which shows the complexation of $\mathrm{Cu}^{2+}$ by ligand PQTSC. The possible structure of the $\mathrm{Cu}^{2+}, \mathrm{Co}^{2+}$, $\mathrm{Ni}^{2+}$ and $\mathrm{Zn}^{2+}$ with PQTSC was shown in scheme 2.

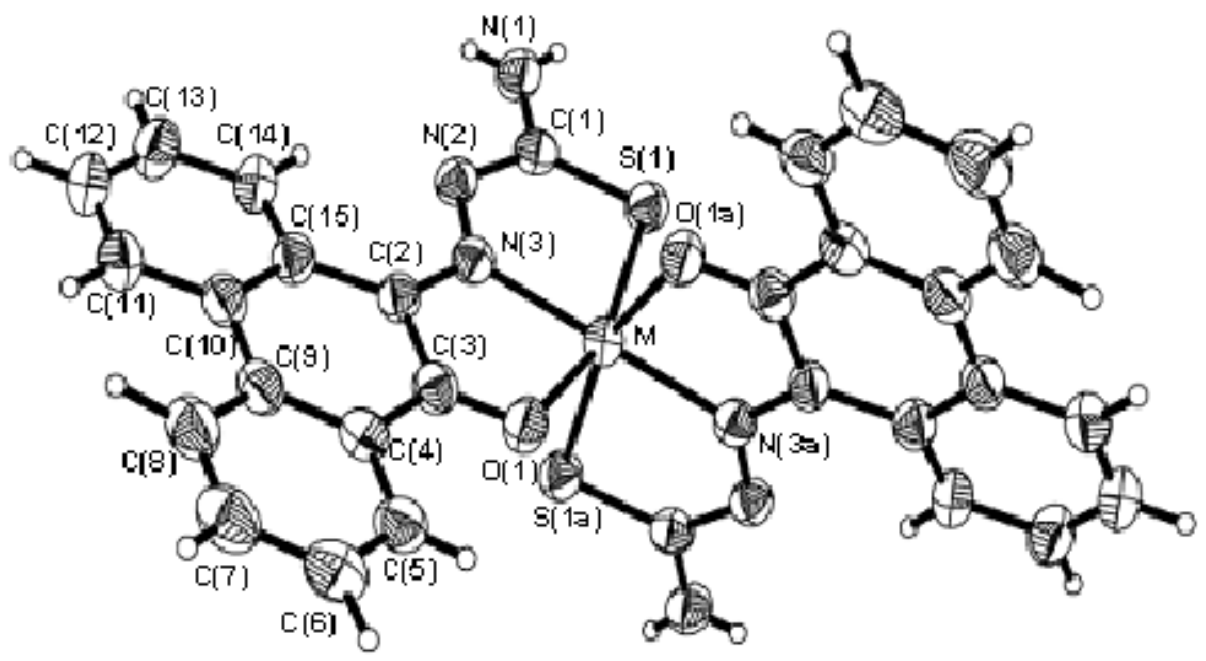

Scheme 2

\section{Results and discussion}

\subsection{Experimental data analysis}

In order to apply model based analysis, the stochimetric and the reactions should be known. Assuming the equation (1-2) for a one step 1:1 complex of ligand with metallic cations and also assuming mass balance equations (3-4) for this reaction, the equation 5 could be derived easily which is the model will be used for model based analysis of such reaction.

$$
\begin{aligned}
& M^{n+}+L^{p-} \stackrel{k_{f 1}}{\longleftrightarrow} M L^{n-p} \\
& k_{f 1}=\frac{\left[M L^{n-p}\right]}{\left[M^{n+}\right]\left[L^{p-}\right]} \\
& C_{L}=\left[L^{p-}\right]+\left[M L^{n-p}\right]
\end{aligned}
$$

and

$C_{M}=\left[M^{n+}\right]+\left[M L^{n-p}\right]$ 
where $C_{L}$ is total ligand and $C_{M}$ is total salt concentration of cation.

$K_{f}[L]^{2}+\left(1+K_{f}\left(C_{M}-C_{L}\right)\right)[L]-C_{L}=0$

This equation has been derived using equations (1-4) with respect to ligand concentration:

Alternatively these equations could be solved so that the derived equation is based on $\left[\mathrm{M}^{\mathrm{n}+}\right]$ or $\left[M L^{n-p}\right]$ concentrations.

Model based analysis is very simple when there is selective wavelength for at least one of the contributed component. In this case the results at selective wavelength were fitted to known model and the formation constant(s) was estimated. But for the cases of highly overlapped spectra of complex (es), ligand and also metallic cations the conventional methods cannot be used to accurate estimation of $\mathrm{K}_{\mathrm{f}}$. But multiwavelength methods are very good alternative in such cases. In this contribution, the multiwavelength model-based analysis method was used to study the formation constant of complexation between several metallic cations with PQTSC regardless of their spectra overlapping.

It should be noted that for the case 1:2 one step $\mathrm{ML}_{2}$ complex formation occurred (Eq. (6) and Eq. (7)), and taking into account the mass balances (Eqs. (8-9)), the Eq. (10) could be derived as ligand concentration:

$$
\begin{aligned}
& M^{n+}+2 L^{p-} \stackrel{k_{f}}{\longrightarrow} M L_{2}^{n-2 p} \\
& k_{f}=\frac{\left[M L_{2}^{n-2 p}\right]}{\left[M^{n+}\right]\left[L^{p-}\right]^{2}} \\
& C_{L}=\left[L^{p-}\right]+2\left[M L_{2}^{n-2 p}\right] \\
& C_{M}=\left[M^{n+}\right]+\left[M L_{2}^{n-2 p}\right] \\
& {[L]^{3}+\left(2 C_{M}-C_{L}\right) K_{f}[L]^{2}+[L]-C_{L}=0}
\end{aligned}
$$

\section{2. Theory of Model-based analysis}

The theory of model-based analysis is available in the literature $[15,17,20]$ and is explained here briefly. Assume a two-way spectrophotometric complexometric data (monitored nc reaction at $\mathrm{n} \lambda$ wavelengths), $\mathbf{D}$, is decomposed into the product of $\mathbf{C}$, containing concentration profiles and $\mathbf{S}$ of absorption spectra:

$$
\mathbf{D}=\mathbf{C} \mathbf{S}^{\mathrm{T}}+\mathbf{E} .
$$

The matrix $\mathbf{E}$ is a collection of the residuals, the difference between the measurement $\mathbf{D}$ and its calculated representation $\mathbf{C S}^{\mathrm{T}}$.

In hard model analysis, the aim is finding the set of parameters according to Eq. (12) so that the sum over all the squares, ssq, over all the elements of the error matrix, $\mathbf{E}$, is minimal ${ }^{20}$.

$$
s s q=\sum \mathbf{E}_{i, j}^{2}=f(\mathbf{D}, \text { model, parameters })
$$

As mentioned the first step and the most difficult task in hard-modeling processes is the establishment of the correct model, e.g. the expected species in equilibrium studies. The knowledge 
and experiences of researcher and also some preliminary resolution of data using soft method of analysis will be useful in finding the correct model.

Evolving factor analysis (EFA) and/or orthogonal projection algorithm (OPA) are very useful method to estimate the right model.

The $2^{\text {nd }}$ and $3^{\text {rd }}$ order equations of 5 and 10 were solved using the "roots" command of MATLAB and therefore the matrix $\mathbf{C}$ (concentration profile) by initial estimation of equilibrium constants was established. Using least squares $\left(\mathbf{S}^{\mathrm{T}}=\mathbf{C}^{+} \mathbf{D}\right.$, where $\mathbf{C}^{+}$is the pseudo-inverse of the matrix $\left.\mathbf{C}\right)$ the spectra of included components were estimated, Then the sum of squares can be defined as a function of only the nonlinear parameters (e.g. the equilibrium constants) as Eq. (13) which has been derived using Eq. (12):

$\operatorname{ssq}=f(\mathbf{k})=\mathbf{D}-\mathbf{C} \mathbf{S}^{\mathbf{T}}=\mathbf{D}-\mathbf{C} \mathbf{C}^{+} \mathbf{D}$,

where $\mathbf{k}$ is the vector of nonlinear parameters, as defined by the chosen model. Any method that performs this minimization task, can be applied. In this work, the Newton-Gauss-LevenbergMarquardt algorithm (NGL/M) ${ }^{20}$ was applied because it is fast, robust and additionally delivers basic statistical analysis of the resulting parameters.

\subsection{Analysis of the experimental data}

The complexometric spectra for the complexation of $\mathrm{Ni}^{2+}$ titrated by ligand PQTSC are shown in Fig. 1. Estimation of chemical component and chemical model are keys points in model based analysis. Visual inspection of spectra and singular value decomposition are frequently used for this purpose.

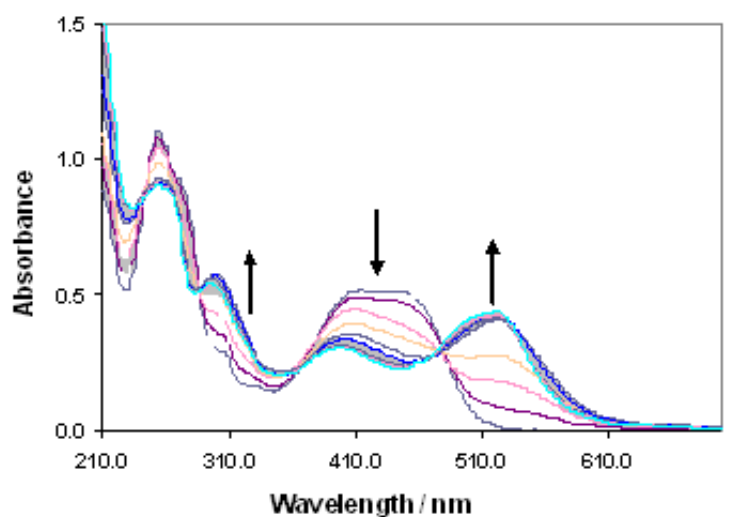

Fig. 1. Changes in UV-Vis absorption spectra of PQTSC in acetonitrile $\left(3.91 \times 10^{-5} \mathrm{~mol} \mathrm{~L}^{-1}\right)$, Arrows indicate a change in the absorbance with increase in the concentration of $\mathrm{Ni}^{2+}$ from a $\left[\mathrm{Ni}^{2+}\right] /[\mathrm{PQTSC}]$ mole ratio of 0.0 to 2.5 .

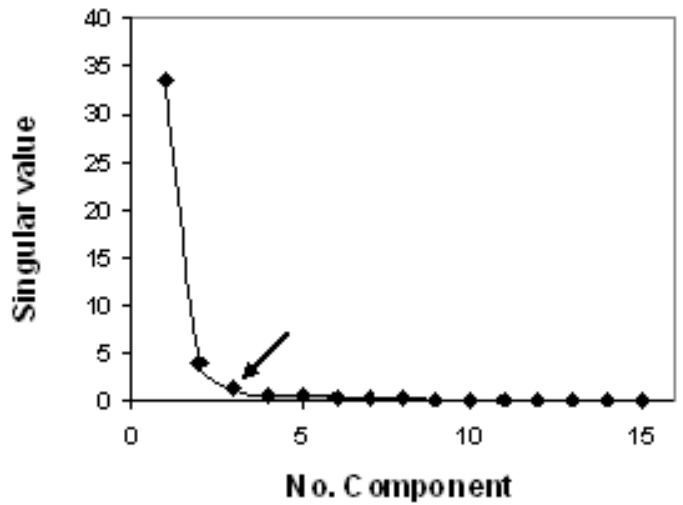

Fig. 2. Consecutive singular values of singular value decomposition of $\mathrm{Ni}^{2+}$-PQTSC complexometric data matrix as a function of number of components.

Preliminary study of this data in the investigated wavelength range 210-610 nm, using singular value decomposition (SVD) showed that there are 3 components (see Fig. 2). It should be noted that $\mathrm{Ni}^{2+}$ and ligand PQTSC are absorbance active in this region. So only one complex, NiL or $\mathrm{NiL}_{2}$, was formed. In order to understand which one of them was formed, the absorbance at $515 \mathrm{~nm}$ was plotted versus ratio $\left[\mathrm{Ni}^{2+}\right] /[\mathrm{PQTSC}]$ (figure is not shown here), it reached the maximum about 0.5 values of that ratio and therefore it can be concluded that that the related system is according to Eq. (6) and the formed complex is $\mathrm{NiL}_{2}$. Therefore absorbing components in this system could be assumed as $\mathrm{L}$, $\mathrm{NiL}_{2}$ and $\mathrm{Ni}^{2+}$ (solvated cation). As mentioned after estimation of correct model, hard model analysis was applied for accurate determination of $\mathrm{K}_{\mathrm{f}}$. In this strategy the concentration profiles and pure spectra of components will be attainable which are the advantages of multiwavlenght model based 
analysis. The extracted concentration profiles and pure spectra of components are shown in Fig. 3 . Using different initial estimates the formation constant of $\mathrm{NiL}_{2}$ complex was calculated to be $10^{11.6}$.

a

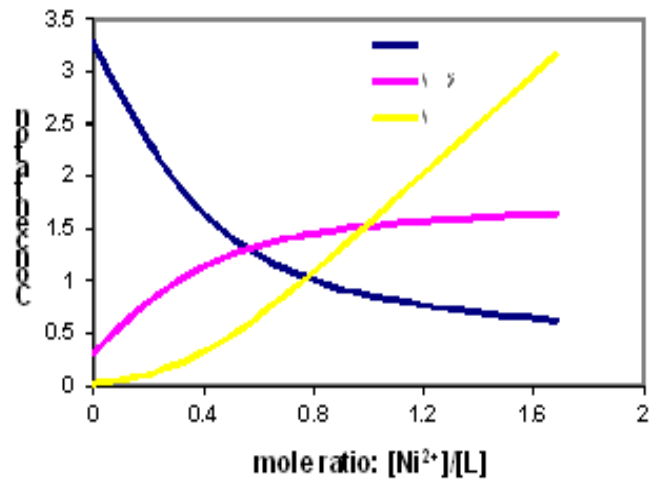

b

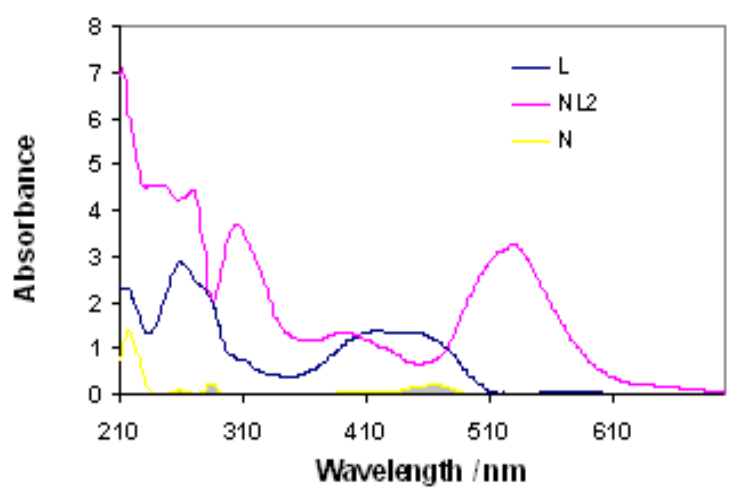

Fig. 3. Results of model based analysis of complexation of $\mathrm{Ni}^{2+}$ with PQTSC in acetonitrile

A same studies was done for the complexation of $\mathrm{Cu}^{2+}, \mathrm{Co}^{2+}$ and $\mathrm{Zn}^{2+}$ with phenanthrenequinone thiosemicarbazone in acetonitrile $\left(\mathrm{Co}^{2+}\right.$ and $\mathrm{Zn}^{2+}$ are not shown). The complexometric spectra for titration $\mathrm{Cu}^{2+}$ of with PQTSC is shown in Fig. 4. The same as previous study SVD was used to estimate the exact number of spectrophotometrically active components. Fig. 5 shows that there are 3 components in this matrix and therefore the model of complexation between $\mathrm{Cu}^{2+}$ and ligand was assumed to be obtained $\mathrm{CuL}_{2}$. The equilibrium constant of $\mathrm{CuL}_{2}$ complex was calculated to be $10^{12.1}$. The resulted concentration profiles and the spectra of involved components in complexometric study of $\mathrm{Cu}^{2+}$ with PQTSC are shown in Fig. 6.

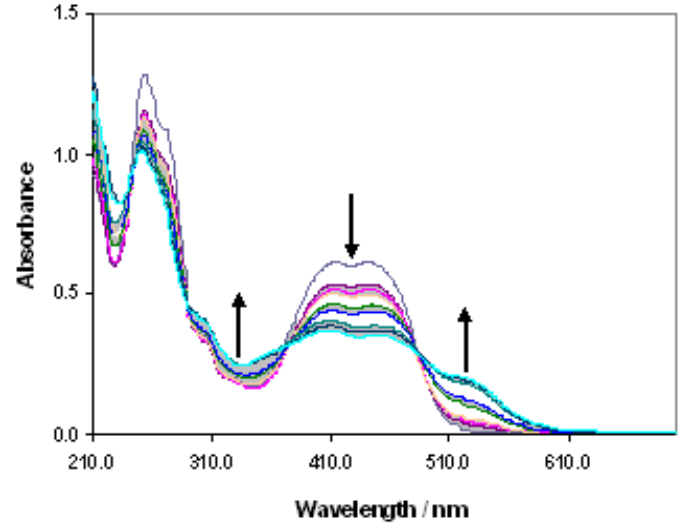

Fig. 4. Changes in UV-Vis absorption spectra of PQTSC in acetonitrile solvent $\left(3.91 \times 10^{-5} \mathrm{~mol} \mathrm{~L}^{-1}\right)$, Arrows indicate a change in the absorbance with increase in the concentration of $\mathrm{Cu}^{2+}$ from a $\left[\mathrm{Cu}^{2+}\right] /[\mathrm{PQTSC}]$ mole ratio of 0.0 to 2.5

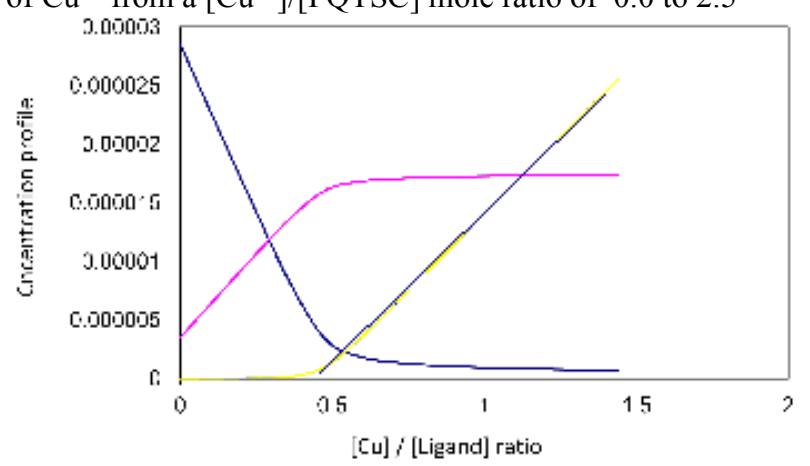

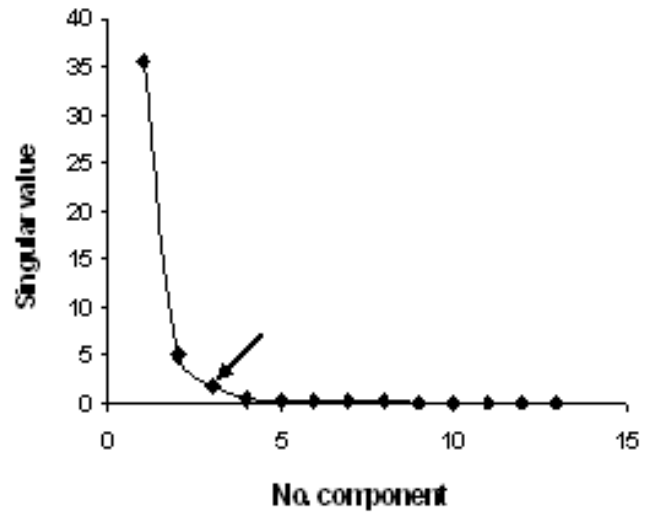

Fig. 5. Consecutive singular values of singular value decomposition of $\mathrm{Cu}^{2+}$-PQTSC complexometric data matrix as a function of number of components

b

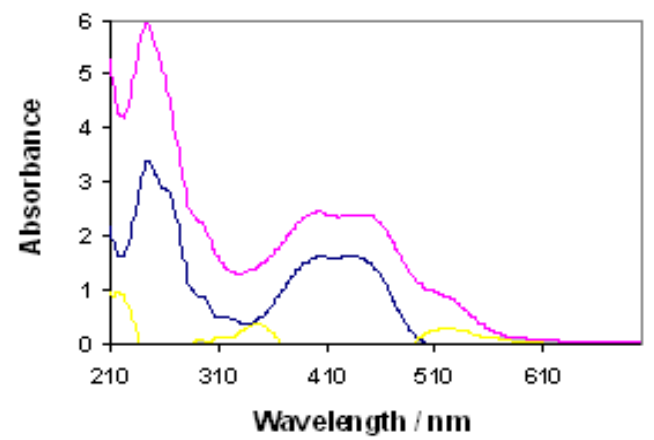

Fig. 6. Results of model based analysis of complexation of $\mathrm{Cu}^{2+}$ with PQTSC in acetonitrile 
Also the stability constants of the complexes $\mathrm{ZnL}_{2}$ and $\mathrm{CoL}_{2}$ were calculated as $10^{9.75}$ and $10^{9.33}$, respectively.

\section{Conclusion}

A hard model analysis method has been used to estimate the formation constants of some metallic complexes with a nearly insoluble synthesized ligand named phenanthrenequinone thiosemicarbazone. The complexes between $\mathrm{Ni}(\mathrm{II}), \mathrm{Co}(\mathrm{II}), \mathrm{Zn}(\mathrm{II})$ and $\mathrm{Cu}(\mathrm{II})$ and this ligand were studied. The results showed that this ligand forms stable complexes with $\mathrm{Cu}(\mathrm{II})$ and $\mathrm{Ni}(\mathrm{II})$. Therefore this ligand can be used be used as a new active probe to detect these cations by absorption spectroscopy and/or can be used for preconcentration and trace analysis of these cations in environmental samples.

\section{Acknowledgement}

We would like to acknowledge Urmia University and University of Tabriz for any support.

\section{References}

1. Powis G.; Hacker M.P. (1991) The Toxicity of Anticancer Drugs, Press: Pergamon, New York.

2. Kappus H. (1986) Overview of enzyme systems involved in bioreduction of drugs and in redox cycling. Biochem. Pharmacol., 35, 1-6.

3. West D.X.; Liberta A.E.; Padhye S.B.; Chikate R.C.; Sonawane P.B.; Kumbhar A.S.; Yerande R.G. (1993) Thiosemicarbazone complexes of copper(II): structural and biological studies. Coordin. Chem. Rev., 123, 49-71.

4. Pierpont C.G.; Buchanan R.M. (1981) Transition metal complexes of o-benzoquinone, osemiquinone and catecholate ligands. Coordin. Chem. Rev., 38, 45-87.

5. Abakumov G.A.; Cherkasov V.K.; Abakumova L.G.; Nevodchikov V.I.; Druzhkov N.O.; Makarenko N.P.; Kursky J.A. (1995) Reaction on the addition of some organometallic compounds to 3,6-di-tert-butyl-o-benzoquinone: new o-quinones. J. Organometal. Chem., 491, 127-133.

6. Saenge W. (1984) Principles of Nucleic Acid Structure. Press: Springer-Verlag, New York.

7. El-Sherif A. A. (2012) potentiometric determination of the stability constants of trimethyltin(iv) chloride complexes with imino-bis(methylphosphonic acid) in water and dioxane-water mixtures. J. Solution Chem., 41, 392-409.

8. Karadag R.; Erdogan G.; Bayar M.; Dolen E. (2007) Determining stability constants of naringenin (4',5,7-trihydroxy flavanone) complexes with aluminium (III) and iron (II) by potentiometric and spectrophotometric methods. Rev. Anal. Chem., 26, 169-186.

9. Saha S.; Tiwary A.; Mukherjee A. (2008) Charge transfer interaction of 4-acetamidophenol (paracetamol) with 2,3-dichloro-1,4-naphthoquinone: A study in aqueous ethanol medium by UVvis spectroscopic and DFT methods. Spectrochim. Acta Part A, 71, 835-840.

10. Rojas-Hernández A.; Botello J.C.; Pacheco-Hernández M.D.L.; Gutiérrez A.; Domínguez J.M.; Espinosa G.; Ramírez-Silva M.T. (2008) Equilibrium constants determination of the species formation in the $\mathrm{Al}(\mathrm{III})-\mathrm{H} 2 \mathrm{O}$ system by integration of 27 . Al-NMR signals and fitting with species fractions. J. Mex. Chem. Soc., 52 (1), 47-53.

11. Zvimb J.; Jackso G. (2007) Thermodynamic and spectroscopic study of the interaction of $\mathrm{Cu}(\mathrm{II}), \mathrm{Ni}(\mathrm{II}), \mathrm{Zn}(\mathrm{II})$ and $\mathrm{Ca}(\mathrm{II})$ ions with 2-amino-N-(2-oxo-2-(2-(pyridin-2-yl)ethyl amino)ethyl) acetamide, a pseudo-mimic of human serum albumin. Polyhedron, 26, 2395-2404.

12. Atabey H.; Sari H. (2011) Potentiometric, theoretical, and thermodynamic studies on equilibrium constants of aurintricarboxylic acid and determination of stability constants of its 
complexes with $\mathrm{Cu}^{2+}, \mathrm{Ni}^{2+}, \mathrm{Zn}^{2+}, \mathrm{Co}^{2+}, \mathrm{Hg}^{2+}$, and $\mathrm{Pb}^{2+}$ metal ions in aqueous solution, J. Chem. Eng. Data, 56 (10), 3866-3872.

13. Ghosh A.K. (2005) Spectrophotometric study of molecular complex formation of asphaltene with two isomeric chloranils. Fuel, 84, 153-157.

14. Tounsi N.; Dupont L.; Mohamadou A.; Aplincourt M.; Plantier-Royon R.; Massicot F.; Harakat D.; Portella C. (2005) Thermodynamic and spectroscopic studies of copper (II) complexes with three bis(amide) ligands derived from L-tartaric acid. J. Inorg. Biochem. 99, 2423-2435.

15. Bahram M.; Pesyan N.N.; Naseri A.; Tasbihforosh M. (2011) Determination of stability constants of 5-(2-hydroxybenzylidene)-2- thioxodihydropyrimidine-4,6 $(1 \mathrm{H}, 5 \mathrm{H})$-dione with copper(II) and mercury(II) ions. Turk. J. Chem., 35(2), 255-264.

16. Hendriksen B.A.; Sanchez-Felix M.V.; Tam K.Y. (2002) A new multiwavelength spectrophotometric method for the determination of the molar absorption coefficients of ionizable drugs. Spectrosc. Lett., 35 (1), 9-19.

17. Meloun M.; Bordovská S.; Syrový T.; Vrána A. (2006) Tutorial on a chemical model building by least-squares non-linear regression of multiwavelength spectrophotometric $\mathrm{pH}$ titration data. Anal. Chim. Acta, 580, 107-121.

18. Emara A.; Saleh A.; Adly O. (2007) Spectroscopic investigations of new binuclear transition metal complexes of Schiff bases derived from 4,6-diacetylresorcinol and 3-amino-1-propanol or 1,3-diamino-propane. Spectrochim. Acta Part A, 68, 592-604.

19. Ghasemi J.; Niazi A.; Maeder M. (2007) Spectrophotometric studies on the protonation and nickel complexation equilibria of 4-(2-pyridylazo) resorcinol using global analysis in aqueous solution, J. Braz. Chem. Soc., 18 (2) , 267-272.

20. Puxty G.; Maeder M.; Hungerbuhler K. (2006) Tutorial on the fitting of kinetics models to multivariate spectroscopic measurements with non-linear least-squares regression, Chemom. Intel. Lab. Syst. 81, 149-164.

21. Kriesten E.; Mayer D.; Alsmeyer F.; Minnich C.B.; Greiner L.; Marquardt W. (2008) Identification of unknown pure component spectra by indirect hard modeling. Chemom. Intel. Lab. Syst. 93, 108-119.

22. Hasani M.; Shariati-Rad M.; Abdollahi H. (2009) Application of soft- and hard-modelling approaches to resolution of kinetics of electron donor-acceptor complex formation of 2,3dichloro-5,6-dicyano-1, 4-benzoquinone with imipramine in different solutions. Anal. Chim. Acta, $636,175-182$.

23. Shariati-Rad M.; Hasani M. (2009) Principle component analysis (PCA) and second-order global hard-modelling for the complete resolution of transition metal ions complex formation with 1,10-phenantroline. Anal. Chim. Acta, 648, 60-70.

24. Mas S.; de Juan A.; Lacorte S.; Tauler R. (2008) Photodegradation study of decabromodiphenyl ether by UV spectrophotometry and a hybrid hard and soft-modelling approach. Anal. Chim. Acta, 618, 18-28.

25. de Juan A.; Maeder M.; Martínez M.; Tauler R. (2000) Combining hard- and soft-modeling to solve kinetic problems. Chemom. Intel. Lab. Syst. 54, 123-141.

26. Afrasiabi Z.; Sinn E.; Padhye Sh.; Dutta S.; Padhye S.; Newton C.; Anson C.; Powelld A.; (2003) Transition metal complexes of phenanthrenequinone thiosemicarbazone as potential anticancer agents: synthesis, structure, spectroscopy, electrochemistry and in vitro anticancer activity against human breast cancer cell-line, T47D. J. Inorg. Biochem. 95, 306-314.

27. West D.X.; Yang Y.; Klein T.L.; Goldberg K.I.; Liberta A.E.; Valdes-Martinez J.; Toscano, R.A. (1995) Binuclear copper(II) complexes of 2-hydroxyacetophenone 4N-substituted thiosemicarbazones. Polyhedron, 14, 1681-1693.

28. West D.X.; Gebremedhin H.; Butcher R.J.; Jasinski J.P.; Liberta A.E. (1993) Structures of nickel(II) and copper(II) complexes of 2-acetylpyridine azacyclothiosemicarbazones. Polyhedron, $12,2489-2497$. 